\title{
Application of two staining methods for sperm morphometric evaluation in domestic pigs
}

\author{
Stanisław Kondracki, Anna Wysokińska, Magdalena Kania, Krzysztof Górski \\ Department of Bioengineering and Animal Breeding, Faculty of Natural Sciences, \\ Siedlce University of Natural Sciences and Humanities, 08-110 Siedlce, Poland \\ annaw@uph.edu.pl
}

Received: May 24, 2017 Accepted: August 23, 2017

\begin{abstract}
Introduction: The effect of two smear staining methods on the dimensions and shape of sperm cells in the semen of domestic pigs was evaluated. Material and Methods: The studies were carried out on 30 ejaculates collected from 15 boars, which included five Duroc boars, five Pietrain boars, and five hybrid Duroc $\times$ Pietrain boars. Each ejaculate was next sampled to make two microscopic slides, of which one was stained with eosin-nigrosin and the other with eosin-gentian dye. In total, 600 measurements of sperm cells were made. Each sperm was measured for the following morphometric parameters: head length, head width, head area, head perimeter, tail length, and the total sperm length. Results: Sperms measured on slides stained with eosin-nigrosin showed lower dimensions as compared with those stained with the eosin-gentian dye method. Sperm stained with eosin-nigrosin had shorter and narrower heads than sperm stained with eosin-gentian dye. The method of staining, therefore, affected not only the dimensions of the sperm, but also the proportions of the dimensions defining the shape of the sperm. Conclusions: The size and shape parameters in porcine sperm may take on different values depending on the method of semen staining. Sperm cells stained with eosin-nigrosin are smaller than the sperm stained with eosin-gentian dye. The sensitivity of the sperm to the type of dye used for the fixation may be associated with genetic factors.
\end{abstract}

Keywords: boars, sperm, morphometry, staining methods.

\section{Introduction}

Male fertility is a key factor in mammalian reproduction and depends on the quality of the produced germ cells. Examination of sperm morphology (24) allows one to diagnose the fertility status in male through determination of sperm structure regularity, revealing any morphological abnormalities. Namely, mammalian sperm cells are characterised by the specific morphological structure that enables them to transfer the genetic information in the process of egg cell fertilisation. It is likely that the ability to penetrate the ovum is related to the size and shape of the spermatozoon. These have been found to vary greatly between species (30), as well as between males of various breeds within the same species (12). Also, considerable differences were found between individuals of the same population (20). A great variation observed in the quality and quantity of porcine semen represents a serious technical and organisational issue in terms of artificial insemination (AI). The factors that change the variability of ejaculate traits include temperature and humidity of the air and atmospheric pressure (30).

The lack of a specific method of semen staining designed for particular species of livestock animals poses a problem as well. Semen tests are carried out using a variety of smear staining methods $(6,21)$. The dyes used are Papanicolaou, eosin-nigrosin, trypan blue, Giemsa, Diff-Quick, and SpermBlue (15, 28). Studies on human sperm revealed that the staining method may significantly influence the results of morphometric measurements (19). The effects of staining on sperm morphometrics have also been found in relation to sperm of bulls, stallions, and rams $(8,17)$.

Papanicolaou staining is time-consuming, multistage, and involves twelve different solutions of chemicals (28). This means that the method has a limited applicability in swine AI stations.

Eosin-nigrosin staining is a popular method for semen evaluation in both mammals and birds $(4,17$, 27). The method enables the analysis of sperm viability and evaluation of its morphological structure. Eosinnigrosin stain has been widely used in boar semen 
analysis, as it is easy to perform and allows detection of morphological abnormalities and determination of cellular membrane integrity. The results of this test are associated with the fertility of sows (4).

Another staining method that has found application in sperm morphology and morphometry evaluation uses the eosin-gentian dye (12). This method is used for staining semen of boars, bulls, and stallions. It is an easy method of staining and it enables accurate observation of respective structures of sperm. Each of these methods may have a different effect on the staining level of particular structures of the sperm as a result of a different response to chemicals which are used. The procedure of staining and agents which are applied can affect the form of sperm's head as well as the whole sperm.

The aim of this study was to evaluate the effect of smear staining method on the dimensions and shape of sperm cells in the semen of domestic pigs.

\section{Material and Methods}

Biological material. The studies were carried out on 30 ejaculates collected from 15 boars, which included 5 Duroc boars, 5 Pietrain boars, and 5 hybrid Duroc $\times$ Pietrain boars. The boars were 18-24 months old. Two ejaculates were collected from each boar by gloved hand once a week. Immediately after collection, the semen was filtered through four layers of sterile gauze into a prewarmed beaker to remove gel particles. The filtered semen was maintained at room temperature until needed for slide preparation for morphometry.

Staining methods. The eosin-nigrosin staining method (differential staining). The preparations for analyses were made according to the following methodology: a drop of semen was placed on a slide preheated to $40^{\circ} \mathrm{C}$ and mixed with double volume of the dye mixture (one part $5 \%$ bluish eosin solution (Carl Roth Gmbh+Co. KG, Germany) to four parts of $10 \%$ nigrosin aqueous solution (Sigma-Aldrich, USA)) using a glass rod to produce a smear on the slide. The samples were air-dried at room temperature.

The eosin-gentian staining method. Thin, fat-free semen smears, heated up to $36^{\circ} \mathrm{C}$, were prepared. After drying, the smears were fixed in $96 \%$ ethanol. Then, they were rinsed with water and counterstained with $10 \%$ blue eosin solution (Carl Roth $\mathrm{Gmbh}+\mathrm{Co} . \mathrm{KG}$, Germany) for 20-60 s. Next the slides were rinsed with water again, and coloured for $3 \mathrm{~min}$ in gentian pigment (Sigma-Aldrich, USA). After colouring, the slides were washed and dried, leading to a clean background and thus to good contrast against the stained spermatozoa. The slides were prepared and assessed microscopically at the same time and by the same person.

Microscopic analysis. All the slides were viewed at a 1,000 magnification under the Nikon E-50i microscope, using immersion objective lenses. Sperm measurements were carried out manually using a computer image analysis package. The images of sperm were examined on the computer screen. In each sample stained with eosin-nigrosin, morphometric measurements involved 10 sperm with a normal cell membrane structure (unstained sperm - viable, Fig. 1).

Similarly, each slide stained with eosin-gentian dye was used to make morphometric measurements of 10 sperm with normal morphology (Fig. 2).

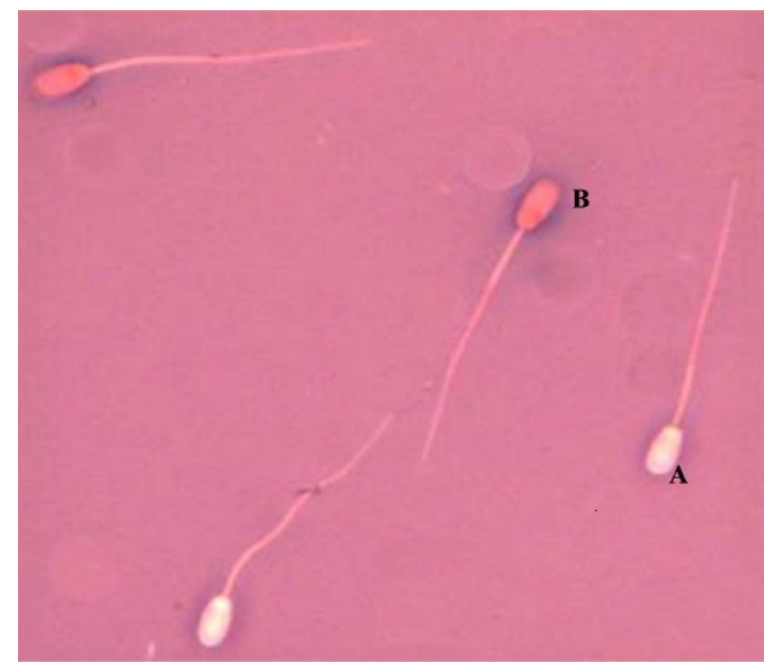

Fig. 1. Specimen stained using eosin-nigrosin (A - unstained sperm cells, viable, B - dead sperm cells with a damaged cell membrane)

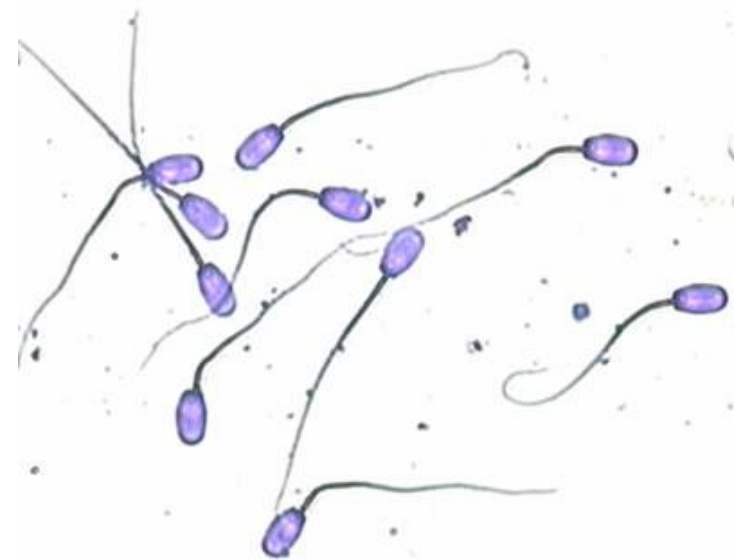

Fig. 2. Sperm cells stained using eosin-gentian dye

In all, 600 measurements of sperm cells were carried out. Each sperm was measured for the following morphometric parameters: head length, head width, head area, head perimeter, tail length, and total sperm length. Based on the measured values, the following ratios of sperm morphology were calculated: head width/head length $\times 100$; head length/sperm length $\times 100$; head length/tail length $\times 100$; tail length/sperm length $\times 100$; head perimeter/sperm length $\times 100$; head area/sperm length $\times 100$; product head length $\times$ width/sperm length $\times 100$.

Statistical analysis. Experimental data were analysed using STATISTICA 10 PL (StatSoft, USA). All results are expressed as mean \pm standard deviation $( \pm$ SD). The obtained material was statistically analysed according to the following mathematical model: $Y_{i j k}=\mu+a_{i}+b_{j}+a b_{i j}+e_{i j k}$, where: $Y_{i j k}-$ value of the analysed parameter, $\mu$ - populational mean, $\mathrm{a}_{\mathrm{i}}$ - staining method effect, $b_{j}-$ effect of individual boars, $a b_{i j}-$ effect of 
cooperation of controlled factors, $\mathrm{e}_{\mathrm{ijk}}$ - error. The significance of the differences between the groups was assessed with the Tukey's test at $\mathrm{P} \leq 0.05$.

\section{Results}

The morphometric data of the sperm stained with eosin-nigrosin and eosin-gentian dyes are presented in Table 1.

Table 1. Boar sperm morphometric parameters by staining method (means $\pm \mathrm{SD}$ )

\begin{tabular}{lcc}
\hline \multirow{2}{*}{ Item } & \multicolumn{2}{c}{ Staining method } \\
\cline { 2 - 3 } & Eosin-nigrosin & Eosin-gentian dye \\
\hline Head length $(\mu \mathrm{m})$ & $8.92^{\mathrm{a}} \pm 0.5$ & $9.31^{\mathrm{b}} \pm 0.47$ \\
\hline Head width $(\mu \mathrm{m})$ & $4.48^{\mathrm{a}} \pm 0.33$ & $4.81^{\mathrm{b}} \pm 0.3$ \\
\hline Head perimeter $(\mu \mathrm{m})$ & $27.69^{\mathrm{a}} \pm 1.85$ & $28.73^{\mathrm{b}} \pm 1.74$ \\
\hline Head area $\left(\mu \mathrm{m}^{2}\right)$ & $33.96^{\mathrm{a}} \pm 3.74$ & $37.43^{\mathrm{b}} \pm 3.74$ \\
\hline Tail length $(\mu \mathrm{m})$ & $44.31^{\mathrm{a}} \pm 2.02$ & $45.17^{\mathrm{b}} \pm 1.66$ \\
\hline Sperm length $(\mu \mathrm{m})$ & $53.24^{\mathrm{a}} \pm 2.18$ & $54.46^{\mathrm{b}} \pm 1.87$ \\
\hline
\end{tabular}

a,b Differences between average values, represented by different letters in the same row, are significant $(\mathrm{P} \leq 0.05)$

The data revealed that the method of smear staining influenced the results of sperm measurements. Sperms measured on slides stained with eosin-nigrosin showed lower dimensions as compared with those fixed on slides stained with the eosin-gentian dye method. Sperm stained with eosin-nigrosin had shorter and narrower heads than sperm stained with eosin-gentian dye $(\mathrm{P} \leq 0.05)$. When stained using eosin-nigrosin, head perimeter was shorter by $1.04 \mu \mathrm{m}$ and head area was smaller by $3.47 \mu \mathrm{m}$ compared to those stained with eosin-gentian dye $(\mathrm{P} \leq 0.05)$. Moreover, sperm stained with eosin-nigrosin had tail shorter by $0.86 \mu \mathrm{m}$ and total sperm length was also shorter by $1.22 \mu \mathrm{m}$, as compared with those stained with eosin-gentian dye $(\mathrm{P} \leq 0.05)$. The differences in the head and tail dimensions of sperm cells, resulting from the type of staining method used, were equally found in relation to Duroc and Pietrain boars, as well as Duroc $\times$ Pietrain crossbreds. The differences were primarily apparent in the head dimensions. However, the effect of smear staining method on the head dimensions was lesser in crossbred boars compared to purebred ones. This was particularly clear when head perimeters (Fig. 3) and head areas (Fig. 4) were compared.

Morphological parameters describing the shape of sperm stained with eosin-nigrosin and eosin-gentian dye are presented in Table 2 . The analysis of the data presented revealed that there were also differences in the shape of sperm stained by eosin-nigrosin and by eosin-gentian dye. The sperm cells stained with eosinnigrosin had more elongated heads than the sperm stained with eosin-gentian dye, as indicated by the head width-to-length ratio of the sperm $(\mathrm{P} \leq 0.05)$. The data in Table 2 also show that the method of staining significantly differentiated other ratios determining the shape of the sperm. For most ratios, lower values were obtained for sperm stained with eosin-nigrosin. Particularly large differences resulting from the staining method were observed in the ratio of head area to sperm length (difference 4.84) and the ratio of the product of head length and width to the length of sperm (difference 7.01). The method of staining therefore affected not only the dimensions of the sperm, but also the proportions of these dimensions, which define the shape of the sperm.

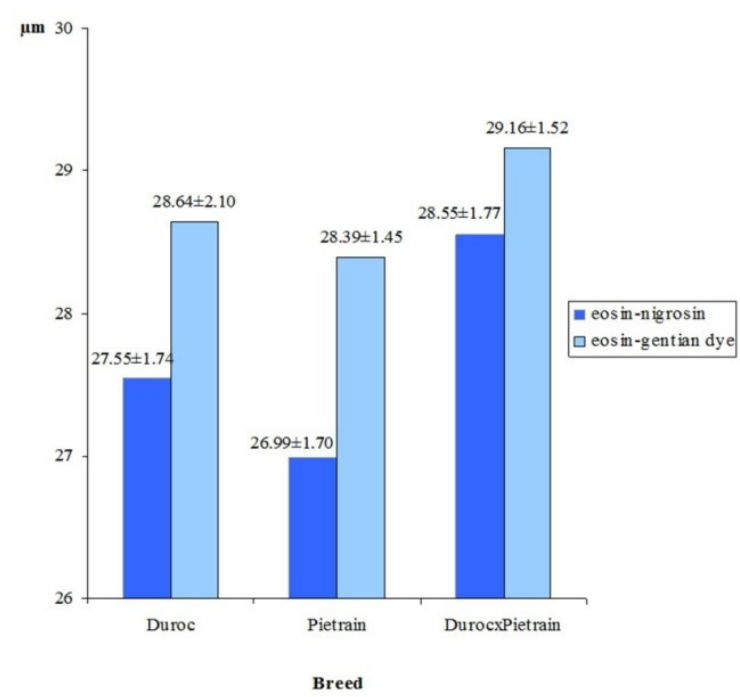

Fig. 3. Sperm head perimeter in Duroc and Pietrain boars and crossbred Duroc $\times$ Pietrain boars in relation to staining method

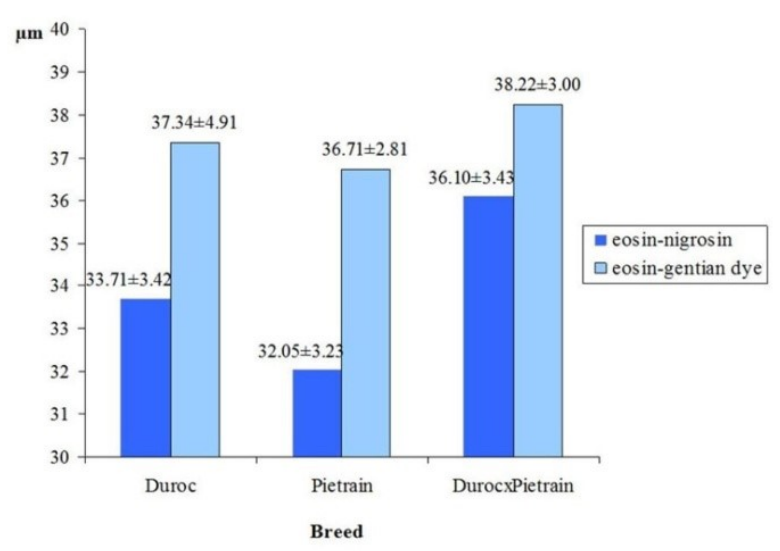

Fig. 4. Sperm head area in Duroc and Pietrain boars and crossbred Duroc $\times$ Pietrain boars in relation to staining method

\section{Discussion}

The data presented here show that smear staining method may have an influence on the results of morphometric measurements of sperm cells, with eosin-nigrosin staining resulting in lower measured dimensions of the sperm compared to eosin-gentian dye staining. The procedure of smear preparation may therefore be important for precision of sperm measurements. Staining techniques may alter sperm dimensions, which had been proven in human semen (19). 
Table 2. Morphological parameters of sperm in relation to staining method (means \pm SD)

\begin{tabular}{lcc}
\hline \multirow{2}{*}{ Item } & \multicolumn{2}{c}{ Staining method } \\
\cline { 2 - 3 } & Eosin-nigrosin & Eosin-gentian dye \\
\hline head width/head length $\times 100$ & $50.36^{\mathrm{a}} \pm 4.43$ & $51.75^{\mathrm{b}} \pm 3.3$ \\
\hline head length/sperm length $\times 100$ & $16.78^{\mathrm{a}} \pm 0.93$ & $17.08^{\mathrm{b}} \pm 0.72$ \\
\hline head length/tail length $\times 100$ & $20.18^{\mathrm{a}} \pm 1.34$ & $20.61^{\mathrm{b}} \pm 1.04$ \\
\hline tail length/sperm length $\times 100$ & $83.22^{\mathrm{a}} \pm 0.93$ & $82.92^{\mathrm{b}} \pm 0.72$ \\
\hline head perimeter/sperm length $\times 100$ & $52.11^{\mathrm{a}} \pm 4.17$ & $52.80^{\mathrm{b}} \pm 3.38$ \\
\hline head area/sperm length $\times 100$ & $63.90^{\mathrm{a}} \pm 7.45$ & $68.74^{\mathrm{b}} \pm 6.48$ \\
\hline product head length $\times$ width/sperm length $\times 100$ & $75.27^{\mathrm{a}} \pm 7.49$ & $82.28^{\mathrm{b}} \pm 6.72$ \\
\hline
\end{tabular}

a,b Differences between average values, represented by different letters in the same row, are significant $(\mathrm{P} \leq 0.05)$

Some staining methods cause sperm cell heads to swell (14), others cause them to shrink (9). Moreover, some smear fixative and staining chemicals were found to affect the size of sperm (25).

This study demonstrates that staining with eosinnigrosin and eosin-gentian dye may produce varied results when it comes to dimensions and shape parameters of domestic pig sperm. Either staining method represents in practice a completely different analytic tool. Eosin-nigrosin method is a negative, differentiating stain (22). It reduces the number of visible artifacts, does not impede the detection of protoplasmic drops, and allows discrimination of live and dead spermatozoa (11). Staining with eosinnigrosin will leave unstained sperm with intact membranes, whereas cells with damaged membranes will be stained (1). A large percentage of stained sperm indicates a reduced fertility of the male. Nigrosin staining produces a dark background, which improves the visibility of sperm cells. Eosin-gentian dye stains all sperm cells purple (both live and dead). The result of the measurements can be affected by the normal morphology of spermatozoon structures. The degree of semen dilution and an adequate proportion between the dye concentration and exposure time of the microscopic slide to the dye are also important factors $(1,4)$.

Sperm cells of various species differ in sensitivity to fixing chemicals (18). Therefore, a proper semen staining method should be selected for a given species, so that changes in the dimensions and shape of sperm cells are as low as possible. In this study, two staining methods for porcine semen morphometric analysis, eosin-nigrosin and eosin-gentian dye, were applied. Eosin-nigrosin is the most commonly used staining method in sperm morphological analysis. It allows detection of live and dead sperm cells (8), and is recommended by the WHO for human semen analysis. This study revealed that porcine spermatozoa stained with eosin-nigrosin had smaller dimensions compared to those stained with eosin-gentian dye. These differences might have resulted from the degree of sperm staining. Studies on staining semen smears of purebred Arabian horses demonstrated that the applied staining method - either eosin-nigrosin or eosin-gentian dye - had little influence on the frequency of the observed morphological abnormalities, and both methods were recommended for equine semen analysis (17). Other studies demonstrated that staining with eosin-nigrosin yields similar results in stallion sperm morphology analysis as Papanicolaou (5).

The size and shape of the head may be related to the motility of the sperm. It has been found that the size of the head and midpiece of porcine spermatozoa are correlated with their motility (10). Spermatozoa with malformed heads are less motile, which may lead to reduced fertilisation rates (10). Some authors claim that the shape of the head is genetically determined (26) and is associated with the changes in the sperm chromatin during the process of spermatogenesis (3). This alters the composition of proteins of the nucleus, whereby histones are converted to protamines. As a result of these changes, sperm chromatin undergoes restructuring and sperm head irregularities or abnormalities may usually appear at this stage. Spermatogenesis and sperm maturation are very dynamic processes in terms of DNA replication and packaging, so any genetic or environmental disturbances may affect the formation of the sperm and lead to morphological abnormalities (7).

Differences in the dimensions of the sperm may result from environmental factors - which include the conditions during semen collection, dilution and storage - but also from genetic factors, or an interaction between these two (16). Temperature, humidity, and air pressure affect the quality of the collected ejaculates (13). Shrinking of sperm heads was observed under low temperatures. Such effects may occur due to changes in osmotic pressure, damage or loss of the acrosome (23) and chromatin condensation in the nucleus of the sperm cell (2). It has also been found that the size and shape of the sperm depend on sperm count in the ejaculate and on the concentration of spermatozoa (29).

In conclusion, the size and shape parameters in porcine sperm may take on different values depending on the method of semen staining. Sperm cells stained with eosin-nigrosin are smaller than the sperm stained with eosin-gentian dye. The heads are shorter and narrower, and the tail is shorter as well. The heads of 
sperm stained with eosin-nigrosin also have a shorter perimeter and a smaller area compared to those stained with eosin-gentian dye. The sensitivity of the sperm to the type of dye used for the fixation may be associated with genetic factors.

Conflict of Interests Statement: The authors declare that there is no conflict of interests regarding the publication of this article.

Financial Disclosure Statement: This study was financed by statutory research and development activity funds from the Polish Ministry of Science and Higher Education assigned to the Faculty of Natural Sciences, Siedlce University of Natural Sciences and Humanities.

Animal Rights Statement: The boars were maintained in accordance with the principles of animal welfare. Approval for this study was granted by the Ethical Committee of Siedlce University of Natural Sciences and Humanities.

\section{References}

1. Almadaly E., Farrag F., Shukry M., Murase T.: Plasma membrane integrity and morphology of frozen-thawed bull spermatozoa supplemented with desalted and lyophilized seminal plasma. Global Vet 2014, 13, 753-766.

2. Álvarez M., Garcia-Macias V., Martinez-Pastor F., Martinez F., Borragán S.: Effects of cryopreservation on head morphometry and its relation with chromatin status in brown bear (Ursus arctos) spermatozoa. Theriogenology 2008, 70, 1498-1506.

3. Andrabi S.M.H.: Mammalian sperm chromatin structure and assessment of DNA fragmentation. J Assist Reprod Genet 2007, 24, 561-569.

4. Björndahl L., SöderlundI., Johansson S., Mohammadieh M., Pourian M.R., Kvist U.: Why the WHO recommendations for eosin-nigrosin staining techniques for human sperm vitality assessment must change. J Androl 2004, 25, 671-678.

5. Brito L.F.C., Greene L.M., Kelleman A., Knobbe M., Turner R.: Effect of method and clinical on stallion sperm morphology evaluation. Theriogenology 2011, 76, 745-750.

6. Cardoso R.C.S., Silva A.R., Silva L.D.M.: Métodos de avaliacão do semen canino congelado. Rev Bras Reprod Anim 2005, 29, 179-187.

7. Enciso M., Cisale H., Johnson S.D., Sarasa J., Fernandez J.L., Gosalvez J.: Major morphological sperm abnormalities in the bull are related to sperm DNA damage. Theriogenology 2011, 76, 23-32.

8. Frenau G.E., Chenoweth P.J., Ellis R., Rupp G.: Sperm morphology of beef bulls evaluated by two different methods. Anim Reprod Sci 2010, 118, 176-181.

9. Gao D.Y., Ashworth E., Watson P.F., Kleinhans F.W., Mazur P., Crister J.K.: Hyperosmotic tolerance of human spermatozoa: separate effects of glycerol, sodium chloride, and sucrose on sperm analysis. Biol Reprod 1993, 49, 112-123.

10. Gil M.C., García-Herreros M., Barón F.J., Aparicio I.M., Santos A.J., García-Marín L.J.: Morphometry of porcine spermatozoa and its functional significance in relation with the motility parameters in fresh semen. Theriogenology 2009, 71, 254-263.

11. Katila T.: In vitro evaluation of frozen-thawed stallion semen: a review. Acta Vet Scand 2001, 42, 199-221.

12. Kondracki S., Iwanina M., Wysokińska A., Huszno M.: Comparative analysis of Duroc and Pietrain boar sperm morphology. Acta Vet Brno 2012, 81, 141-145.
13. Kowalewski D., Kondracki S., Górski K., Bajena M., Wysokińska A.: Effect of piggery microclimate on ejaculate performance of artificial insemination boars. Kafkas Univ Vet Fak Derg 2016, 22, 225-232.

14. Kruger T.F., Ackerman S.B., Simmons K.F., Swanson R.J., Brugo S.S., Acosta A.A.: A quick, reliable staining technique for human sperm morphology. Arch Androl 1987, 18, 275-277.

15. Kruger T.F., Lacquet F.A., Sarmiento C.A., Menkveld R., Ozgur K., Lombard C.J., Franken D.R.: A prospective study on the predictive value of normal sperm morphology as evaluated by computer (IVOS). Fertil Steril 1996, 66, 285-291.

16. Lavara R., Vincente J.S., Baselga M.: Genetic variation in head morphometry of rabbit sperm. Theriogenology 2013, 80, 313-318.

17. Lącka K., Kondracki S., Iwanina M., Wysokińska A.: Assessment of stallion semen morphology using two different staining methods, microscopic techniques, and sample sizes. J Vet Res 2016, 60, 99-104.

18. Łukaszewicz E., Jerysz A., Partyka A., Siudzińska A.: Efficacy of evaluation of rooster sperm morphology using different staining methods. Res Vet Sci 2008, 85, 583-588.

19. Maree L., du Plessis S.S., Menkveld R., van der Horst G.: Morphometric dimensions of the human sperm head depend on the staining method used. Hum Reprod 2010, 25, 1369-1382.

20. Maroto-Morales A., Ramón M., García-Álvarez O., Soler A.J., Esteso M.C., Martinez-Pastor F., Pérez-Guzmán M.D., Garde J.J.: Characterization of ram (Ovis aries) sperm head morphometry using the Sperm-Class Analyzer. Theriogenology 2010, 73, 437-448

21. Oberlender G., Murgas L.D.S., Zangeronimo M.G., Silva A.C., Pereira L.J., Muzzi R.A.L.: Comparison of two different methods for evaluating boar semen morphology. Arch Med Vet 2012, 44, 201-205.

22. O'Connel M., McClure N., Lewis S.E.: The effect of cryopreservation on sperm morphology, motility, and mitochondrial function. Hum Reprod 2002, 17, 704-709.

23. Peña A.I., Lugilde L.L., Barrio M., Herradon P.G., Quintela L.A.: Effects of Equex from different sources on post-thaw survival, longevity and intracellular $\mathrm{Ca}_{2+}$ concentration of $\operatorname{dog}$ spermatozoa. Theriogenology 2003, 59, 1725-1739.

24. Phillips N.J., McGowan M.R., Johnston S.D., Mayer D.G.: Relationship between thirty post-thaw spermatozoal characteristics and the field fertility of 11 high-use Australian dairy AI sires. Anim Reprod Sci 2004, 81, 47-61.

25. Sancho M., Perez-Sanchez F., Tablado L., de Monserrat J.J., Soler C.: Computer assisted morphometric analysis of ram sperm heads: evaluation of different fixative techniques. Theriogenology 1998, $50,27-37$.

26. Thurston L.M., Watson P.F., Holt W.V.: Sources of variation in the morphological characteristics of sperm subpopulations assessed objectively by a novel automated sperm morphology analysis system. J Reprod Fertil 1999, 117, 271-280.

27. van der Horst G., Kitchin R.M., van der Horst M., Atherton R.W.: The effect of the breeding season, cryopreservation and physiological extender on selected sperm and semen parameters of four ferret species: implications for captive breeding in the endangered black-footed ferret. Reprod Fertil Dev 2009, 21, 351-363.

28. van der Horst G., Maree L.: SpermBlue: a new universal stain for human and animal sperm which is also amenable to automated sperm morphology analysis. Biotech Histochem 2009, 84, 299-308.

29. Wysokińska A., Kondracki S., Banaszewska D.: Morphometrical characteristics of spermatozoa in Polish Landrace Boars with regard to the number of spermatozoa in an ejaculate. Reprod Biol 2009, 9, 271-282.

30. Yaniz J.L., Soler C., Santolaria P.: Computer assisted sperm morphometry in mammals: a review. Anim Reprod Sci 2015, $156,1-12$. 